\title{
Short Low-Rate Non-Binary Turbo Codes
}

\author{
Gianluigi Liva, Balázs Matuz, Enrico Paolini, Marco Chiani
}

\begin{abstract}
A serial concatenation of an outer non-binary turbo code with different inner binary codes is introduced and analyzed. The turbo code is based on memory- 1 time-variant recursive convolutional codes over high order fields. The resulting codes possess low rates and capacity-approaching performance, thus representing an appealing solution for spread spectrum communications. The performance of the scheme is investigated on the additive white Gaussian noise channel with coherent and noncoherent detection via density evolution analysis. The proposed codes compare favorably w.r.t. other low rate constructions in terms of complexity/performance trade-off. Low error floors and performances close to the sphere packing bound are achieved down to small block sizes ( $k=192$ information bits).
\end{abstract}

\section{INTRODUCTION}

Low-rate codes have been widely considered in the context of spread spectrum communications [1], [2]. Some of the most successful and powerful coding schemes are based on Hadamard-Walsh sequences either for orthogonal modulation [2], [3] or as component codes for concatenated schemes [2], [4]-[6]. For instance, a low-rate coding scheme consisting of the concatenation of an outer rate- $1 / 3$ convolutional code with an inner Hadamard code, leading to a coding rate of $1 / 32$, was selected for the uplink of the IS-95(A) standard [2], [3].

Iteratively-decodable codes able to approach the Shannon limit at low coding rates have been introduced in the past [6]-[8]. However, most of them suffer either from high error floors [6], [7] or from visible losses compared with the sphere packing bound (SPB) [9] when the code dimension $k$ is within few hundreds of bits [8]. Very low-rate low-density paritycheck (LDPC) codes over moderate order fields $\mathbb{F}_{q}$ (e.g., with $q=2^{m}, 6 \leq m \leq 8$ ) possessing decoding thresholds close to channel capacity have been recently proposed in [10]. The codes of [10], also referred to as multiplicative repeat (MR)LDPC codes, rely on the repetition of the codeword symbols and their multiplication by non-zero coefficients of $\mathbb{F}_{q}$. They can be described as the serial concatenation of an outer LDPC code over $\mathbb{F}_{q}$ and binary inner codes with dimension $m$.

In this paper, a novel low-rate scheme is presented based on the concatenation of inner algebraic codes having good distance properties with the recently-introduced non-binary turbo codes of [11] as outer codes where the inner code dimension matches the turbo code field order $q$. The proposed concatenation can in principle be also applied to ultra-sparse non-binary LDPC codes. The concatenated code performance is first analyzed by means of density evolution (DE) for both coherent and noncoherent detection, showing how their

G. Liva and B. Matuz are with Institute of Communications and Navigation of the Deutsches Zentrum für Luft- und Raumfahrt (DLR), 82234 Wessling, Germany (e-mail: \{gianluigi.liva, balazs.matuz\}@dlr.de).

E. Paolini and M. Chiani are with CNIT, DEIS/WiLAB, University of Bologna, 47521 Cesena (FC), Italy (e-mail: \{e.paolini, marco.chiani\}@unibo.it). decoding thresholds lie within $0.5 \mathrm{~dB}$ from the Shannon limit in the coherent case, for a wide range of coding rates $(1 / 3 \leq R \leq 1 / 96)$. Remarkably, a similar result is achieved in the noncoherent detection framework as well.

We then focus on the specific case where the inner code is either an order- $q$ Hadamard code or a first order length- $q$ ReedMuller (RM) code, due to their simple fast Hadamard transform (FHT)-based decoding algorithms. The proposed scheme can be thus seen either as (i) a serial concatenation of an outer $\mathbb{F}_{q}$-based turbo code with an inner Hadamard/RM code with antipodal signalling, or (ii) as a coded modulation $\mathbb{F}_{q}$-based turbo/LDPC code with $q$-ary (bi-) orthogonal modulation. The soft demodulation and the non-binary trellis/check node soft-input soft-output (SISO) blocks can be both efficiently implemented thanks to the order- $q$ FHT. This allows full hardware reuse at the decoder. The proposed construction performs within $0.8 \mathrm{~dB}$ from the SPB in the short block regime over an additive white Gaussian noise (AWGN) channel with coherent detection. Remarkably, low error floors are achieved. We further compare the obtained performance with that of the IS-95(A) standard with iterative demodulation/decoding [12], observing gains of $2 \mathrm{~dB}$ or more. We also simulate the concatenated scheme performance on the AWGN with noncoherent detection, for which the phase of the channel is assumed to be blockwise constant [13]. Again a large gain w.r.t. IS-95(A) standard can be observed.

\section{Code Structure And Decoding}

We consider an $\left(n_{\circ}, k_{0}\right)$ outer code $\mathscr{C}_{0}$ over $\mathbb{F}_{q}$, with $q=2^{m}$, where $n_{\circ}$ and $k_{\circ}$ denote the block length and the code dimension in terms of field symbols. The coding rate is given by $R_{\circ}=k_{\circ} / n_{\circ}$. Due to their excellent performance in the short block length regime, we restrict our analysis to outer codes being non-binary turbo codes based on memory-1 recursive convolutional codes [11], for which iterative decoding can be efficiently implemented thanks to FHTs [14] with complexity $\mathcal{O}(q \log q)$. The encoder structure is depicted in Figure 1. The information word $\mathbf{u}$ of $k_{\mathrm{O}}$ symbols in $\mathbb{F}_{q}$ is input to a rate-1, memory-1 time-variant recursive systematic convolutional (RSC) tail-biting encoder. The first set of parity symbols $\mathbf{p}^{(1)}$ is obtained as

$$
p_{i}^{(1)}=g_{i}^{(1)} u_{i}+f_{i}^{(1)} p_{i-1}^{(1)} \quad \forall i \in\left[0, k_{\circ}-1\right],
$$

with $p_{-1}^{(1)}=p_{k_{0}-1}^{(1)}$ properly initialized. Here, $p_{i}^{(1)} \in \mathbb{F}_{q}$ and $g_{i}^{(1)}, f_{i}^{(1)} \in \mathbb{F}_{q} \backslash\{0\}$. The second set of parity symbols $\mathbf{p}^{(2)}$ is obtained in a similar way after permuting the symbols of $\mathbf{u}$ according to the interleaving rule $i \mapsto \pi(i)$ (for details see [11]). The codeword is given by $\mathbf{w}=\left[\mathbf{u}\left|\mathbf{p}^{(1)}\right| \mathbf{p}^{(2)}\right]$. The code length is $n_{\circ}=3 k_{\circ}$ symbols and the coding rate is $R_{\bigcirc}=1 / 3$. 


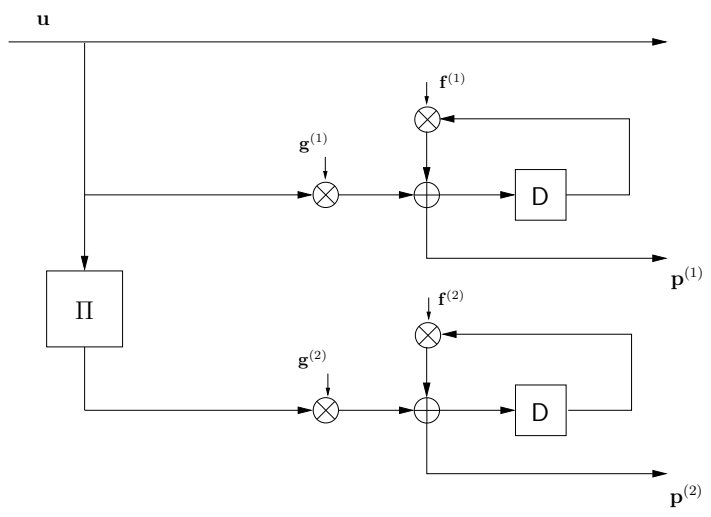

Fig. 1. Encoder for the non-binary turbo codes of [11].

The non-binary turbo code is serially-concatenated [15] with an inner $\left(n_{1}, k_{1}\right)$ binary linear block code $\mathscr{C}_{1}$, where both block length and code dimension are expressed in bits. For the proposed concatenated scheme it is assumed that $k_{\mathrm{I}}=m$. The coding rate of the inner code is $R_{\mathrm{l}}=k_{\mathrm{l}} / n_{\mathrm{l}}$. Since each symbol at the output of the outer encoder is mapped onto a codeword of $\mathscr{C}_{1}$, the overall block length in bits is $n=n_{\mathrm{O}} \times n_{1}$. The overall rate for the concatenated code $\mathscr{C}$ is $R=R_{\mathrm{O}} \times R_{1}=k / n$, where $k=k_{\mathrm{O}} \times m$ is the input block size of the outer turbo code in bits. Note that $\mathscr{C}$ is linear upon proper choice of the mapping $C_{1}(\beta): \mathbb{F}_{q} \mapsto \mathscr{C}_{1}$ between $\mathbb{F}_{q}$ symbols and codewords in $\mathscr{C}_{1}$. More specifically, linearity is achieved by multiplying the $m$-bits binary vector representation of the encoded symbols by the generator matrix of the inner code. The minimum distance for the concatenated code $\mathscr{C}$ is $d \geq d_{\circ} \times d_{1}$, where $d_{0}$ and $d_{1}$ are the minimum distances of outer and inner codes.

Let's denote by $\mathbf{w}=\left[\begin{array}{llll}w_{0} & w_{1} & \ldots & w_{n_{\circ}-1}\end{array}\right]$ the $n_{0}$-symbols codeword of the outer turbo code and by $\mathbf{c}$ the codeword at the output of the concatenated encoder. Then, $\mathbf{c}$ can be partitioned into $n_{\circ}$ segments of $n_{1}$ bits each, $\mathbf{c}=\left[\begin{array}{llll}\mathbf{c}_{0} & \mathbf{c}_{1} \ldots & \ldots & \mathbf{c}_{n_{\circ}-1}\end{array}\right]$, where the generic $t$-th segment $\mathbf{c}_{t}$ is associated to the $t$-th symbol at the output of the turbo encoder. Clearly, $\mathbf{c}_{t} \in \mathscr{C}_{1}$. The bits of $\mathbf{c}$ are finally mapped onto a binary antipodal modulation, producing the modulated vector $\mathbf{x}=1-2 \mathbf{c}$. As for $\mathbf{c}$, also $\mathbf{x}$ is partitioned into $n_{\circ}$ segments, $\mathbf{x}=\left[\begin{array}{llll}\mathbf{x}_{0} & \mathbf{x}_{1} & \ldots & \mathbf{x}_{n_{\circ}-1}\end{array}\right]$.

\section{A. Coherent Detection}

We first consider transmission over the AWGN channel under the assumption of coherent detection. The received signal $\mathbf{y}$ is given by

$$
\mathbf{y}=\mathbf{x}+\mathbf{n}
$$

with $n_{i} \sim \mathcal{N}\left(0, \sigma^{2}\right)$. Also for $\mathbf{y}$ and $\mathbf{n}$ we adopt the same partitioning of $\mathbf{c}$ and $\mathbf{x}$, with $\mathbf{y}_{t}$ denoting the channel output for $\mathbf{x}_{t}$ and $\mathbf{n}_{t}$ the corresponding noise samples. Decoding is performed in two stages. For each received segment $\mathbf{y}_{t}$, the conditional probability mass function $(\mathrm{PMF}) P_{t}(\beta)$ is evaluated for each $\beta \in \mathbb{F}_{q}$, where

$$
P_{t}(\beta):=\operatorname{Pr}\left\{w_{t}=\beta \mid \mathbf{y}_{t}\right\}
$$

represents the probability that the symbol associated with $\mathbf{y}_{t}$ is $\beta$, given the observation of $\mathbf{y}_{t}$. Due to the mapping $\mathrm{C}_{1}(\beta): \mathbb{F}_{q} \mapsto \mathscr{C}_{1},(2)$ can be rewritten as $P_{t}(\beta)=\operatorname{Pr}\left\{\mathbf{x}_{t}=\right.$ $\left.\mathbf{x}_{t}^{(\beta)} \mid \mathbf{y}_{t}\right\}$, where $\mathbf{x}_{t}^{(\beta)}=1-2 \mathbf{c}_{t}^{(\beta)}$ and $\mathbf{c}_{t}^{(\beta)}=\mathrm{C}_{\mathrm{l}}(\beta)$. Under the assumption of $\beta$ uniformly distributed over $\mathbb{F}_{q}$, the PMF $P_{t}(\beta)$ fulfills

$$
P_{t}(\beta) \propto \exp \left(\frac{1}{\sigma^{2}}\left\langle\mathbf{x}_{t}^{(\beta)}, \mathbf{y}_{t}\right\rangle\right)
$$

where $\left\langle\mathbf{x}_{t}^{(\beta)}, \mathbf{y}_{t}\right\rangle$ denotes the inner product (correlation) between $\mathbf{x}_{t}^{(\beta)}$ and $\mathbf{y}_{t}$. The PMFs $P_{t}(\beta), t=0, \ldots, n_{\circ}-1$, are then input to the iterative decoder operating on the factor graph for the outer turbo code. Note that usually, when binary outer codes are employed, a marginalization is performed after computing the channel conditional probabilities to derive bitwise probabilities [12]. This leads to a loss of information that may be partially recovered by iterating decoding between the inner and the outer code [12]. Alternatively, symbol-based decoding of the outer code may be performed by merging sections of its trellis representation [16], thus avoiding the need of marginalizing the probabilities $P_{t}(\beta)$. This allows skipping the iteration between the outer and the inner decoder, at the expense of a higher outer code decoding complexity. We will see that the proposed concatenated scheme, despite working symbol-wise, allows keeping a relatively-simple outer decoder, with a complexity lower than that of [16].

\section{B. Noncoherent Detection}

We consider next a blockwise noncoherent channel with AWGN [13]. We assume the phase to be constant over blocks of $n_{1}$ channel bits, i.e., over each inner code word. The received signal associated with the $t$-th turbo code symbol is

$$
\mathbf{y}_{t}=\mathbf{x}_{t} e^{j \theta_{t}}+\mathbf{n}_{t} .
$$

The noise samples are modeled as complex, circularlysymmetric Gaussian random variables, $\mathcal{C N}\left(0,2 \sigma^{2}\right)$ and $\theta_{t}$ is uniformly-distributed, $\theta_{t} \sim \mathcal{U}[0,2 \pi[$. We further assume the phases of different blocks to be independent. For each $\mathbf{y}_{t}$, the conditional PMF $P_{t}(\beta)$ is evaluated for each $\beta \in \mathbb{F}_{q}$. Due to the mapping $C_{1}(\beta): \mathbb{F}_{q} \mapsto \mathscr{C}_{1}$ and averaging over the distribution of $\theta_{t}$, (2) can be rewritten as

$$
P_{t}(\beta)=\frac{1}{2 \pi} \int_{0}^{2 \pi} \operatorname{Pr}\left\{\mathbf{x}_{t}^{(\beta)} \mid \mathbf{y}_{t}, \theta_{t}\right\} d \theta_{t} .
$$

Under the assumption of $\beta$ uniformly distributed over $\mathbb{F}_{q}$, the PMF $P_{t}(\beta)$ can be easily evaluated as [12]

$$
P_{t}(\beta) \propto \mathrm{I}_{0}\left(\frac{1}{\sigma^{2}}\left|\left\langle\mathbf{x}_{t}^{(\beta)}, \mathbf{y}_{t}\right\rangle\right|\right)
$$

where $I_{0}$ is the modified Bessel function of first kind and order zero.

\section{Code Design And Density Evolution Analysis}

For a given target coding rate, the choice of the inner code shall be based on the concatenated code minimum distance and on the iterative decoding threshold of the overall scheme. To achieve large minimum distances, inner codes 


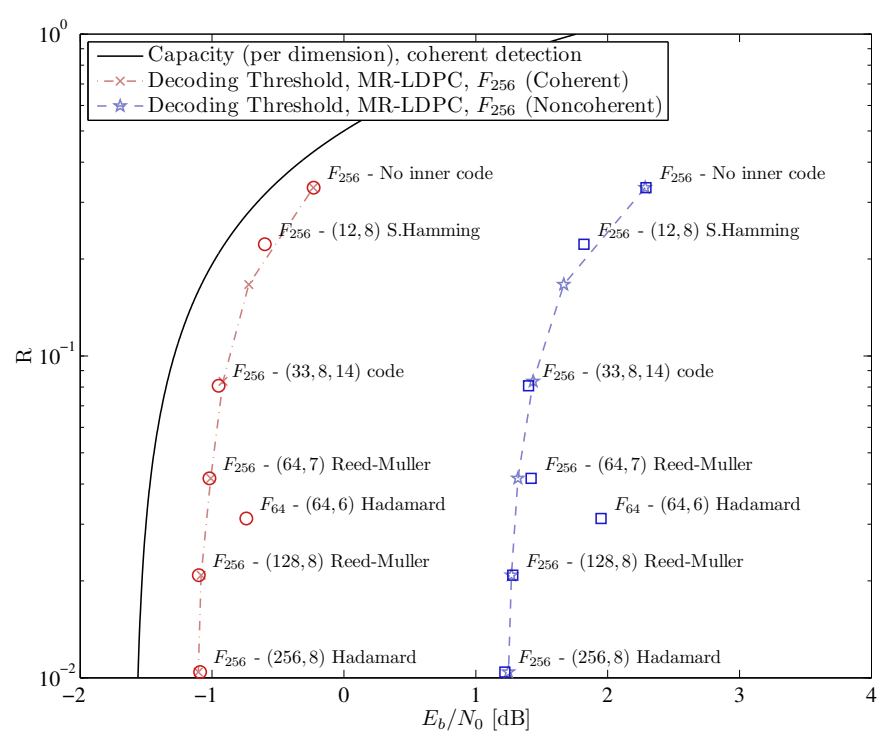

Fig. 2. Iterative decoding thresholds for various concatenations. The 'o' marker denotes the coherent detection case, whereas the ' $\square$ ' marker refers to the noncoherent detection case. The decoding thresholds for the MR-LDPC ensemble over $\mathbb{F}_{256}$ are provided as reference.

with good distance properties will be considered. Next we provide a DE analysis based on the Monte Carlo method for selected combinations of outer and inner codes. The decoding thresholds are evaluated in terms of $E_{b} / N_{0}$, where $E_{b}$ denotes the energy per information bit and $N_{0}$ is the one-sided noise power spectral density. The inner codes that have been selected for the DE analysis are Hamming, Hadamard and RM codes. In some cases, shortening has been applied to fit the inner code dimension to the outer code field size. We additionally considered a large minimum distance code with dimension tailored to the turbo code field $\mathbb{F}_{256}$, i.e. the $(33,8)$ code of [17] with minimum distance 14.

Figure 2 reports the decoding thresholds for different concatenations, under coherent and noncoherent detection. For the coherent detection case, the unconstrained-input channel capacity is also depicted. Considering an outer turbo code on $\mathbb{F}_{256}$, code rates from $1 / 3$ to $1 / 96$ are obtained by selection of different inner codes (no inner code is used for $R=1 / 3$ ). As it can be seen the gap to capacity is within $\sim 0.5 \mathrm{~dB}$. For comparison, the decoding thresholds of the MR-LDPC codes of [10] are also provided for different coding rates. The results nearly match with those of the concatenated scheme. However, as it will be discussed in the next section, when restricting to Hadamard and RM inner codes, the decoding complexity of the proposed solution is lower than that of [10]. We also provide a result on an $\mathbb{F}_{64}$ turbo code in concatenation with the $(64,6)$ Hadamard code. We can observe that by lowering the field order $q$ the gap to capacity increases.

A similar analysis has been performed for the noncoherent detection case. As Figure 2 reports, the iterative decoding thresholds are shifted by $\sim 2.4 \mathrm{~dB}$ for all codes in comparison with the coherent detection case. Only the $\mathbb{F}_{64}$ turbo code in concatenation with the $(64,6)$ Hadamard code is affected by a larger loss, that is around $2.7 \mathrm{~dB}$. Nevertheless, the results are quite promising considering the no phase information is available at the receiver. Note that the decoding threshold under noncoherent detection for the turbo code ensemble over $\mathbb{F}_{256}$ is at $E_{b} / N_{0} \simeq 2.28 \mathrm{~dB}$. We computed the normalized average mutual information (AMI) $I_{N}$ for a blockwise noncoherent channel with phase constant over blocks of $n_{1}=8$ symbols, with the constraint of antipodal mapping,

$$
I_{N}:=\frac{I\left(\mathbf{x}_{t} ; \mathbf{y}_{t}\right)}{n_{\mathrm{l}}}=\frac{1}{n_{\mathrm{l}}} \mathrm{E}\left[\log _{2} \frac{p\left(\mathbf{y}_{t} \mid \mathbf{x}_{t}\right)}{p\left(\mathbf{y}_{t}\right)}\right],
$$

where

$$
\begin{aligned}
& p\left(\mathbf{y}_{t} \mid \mathbf{x}_{t}\right)= \\
& \quad \frac{1}{\left(2 \pi \sigma^{2}\right)^{n_{1}}} \exp \left(-\frac{\left\|\mathbf{x}_{t}\right\|^{2}}{2 \sigma^{2}}-\frac{\left\|\mathbf{y}_{t}\right\|^{2}}{2 \sigma^{2}}\right) \mathrm{I}_{0}\left(\frac{1}{\sigma^{2}}\left|\left\langle\mathbf{x}_{t}, \mathbf{y}_{t}\right\rangle\right|\right) .
\end{aligned}
$$

Under the assumption of $\mathbf{x}_{t}$ distributed uniformly over $\{ \pm 1\}^{n_{1}}$, $I_{N}$ equals $R=1 / 3$ for $E_{b} / N_{0} \simeq 2.06 \mathrm{~dB}$. Remarkably, the rate- $1 / 3$ scheme possesses a decoding threshold that is only $\sim 0.22 \mathrm{~dB}$ away from the limit given by (6). Similarly, the limit provided by (6) for coding rate $R=1 / 24$ and $n_{1}=64$ is at $E_{b} / N_{0} \simeq 0.84 \mathrm{~dB}$, while the decoding threshold for the rate $R=1 / 24$ scheme in Figure 2 is almost at $E_{b} / N_{0} \simeq 1.42$, only $0.6 \mathrm{~dB}$ away.

\section{Turbo Codes over High Order Fields With ORTHOGONAL AND BI-ORTHOGONAL MODULATION}

We focus on the concatenation with Hadamard and first order RM codes for two compelling reasons. First, they achieve low coding rates with performance close to capacity, as emphasized by the DE analysis. Second, Hadamard and first order RM codes can be decoded via FHT. This allows an efficient implementation of the inner decoder, also with the possibility of reusing of the hardware employed by the turbo decoder. Hadamard codes and first order RM codes with antipodal modulation are examples of orthogonal and biorthogonal codes, respectively [3]. Therefore, they lead to the same error probabilities of any other (bi-) orthogonal signal set of the same order, such as pulse position modulation (PPM) and bi-orthogonal PPM. In the following we will refer to Hadamard and first order RM codes as orthogonal and biorthogonal codes to emphasize that the achieved results hold in general when (bi-) orthogonal modulations are used. A derivation of the decoding complexity of the proposed scheme is provided next, followed by a discussion on how the overall coding rate of the scheme can be flexibly adjusted.

\section{A. On the Decoding Complexity}

The antipodal representation of an order- $2^{m}$ Hadamard code can be obtained as follows. Starting from the order- 2 Hadamard matrix,

$$
\mathcal{H}_{2}=\left[\begin{array}{ll}
+1 & +1 \\
+1 & -1
\end{array}\right]
$$

the order- $2^{m}$ Sylvester-type Hadamard matrix is obtained by iterating the Kronecker product [18, Ch. 14]

$$
\mathcal{H}_{2^{m}}=\mathcal{H}_{2} \otimes \mathcal{H}_{2^{m-1}} .
$$


The order- $2^{m}$ Hadamard code modulated sequences correspond to the rows of $\mathcal{H}_{2^{m}}$. The first order, length- $2^{m}$ RM code modulated sequences correspond to the rows of the matrix

$$
\mathcal{H}_{\mathrm{RM}}=\left[\begin{array}{c}
\mathcal{H}_{2^{m}} \\
-\mathcal{H}_{2^{m}}
\end{array}\right] \text {. }
$$

Thanks to this structure, the correlation of (3) and (5) can be evaluated with a complexity that is $\mathcal{O}(q \log q)$, with $q=2^{m}$ via an order- $q$ FHT [18, Ch. 14]. This feature is even more appealing, considering that the forward-backward algorithm over the component trellises of the turbo codes of [11] can be performed efficiently with complexity $\mathcal{O}(q \log q)$. This is again due to the order- $q$ FHT which is used to dualize the check node message passing rule [11]. Thus, when order$q$ Hadamard codes or first order length- $q$ RM codes are employed as outer codes, the overall decoding complexity is $\mathcal{O}(q \log q)$. Furthermore, being the FHT employed in the forward-backward algorithm over the component trellises, an efficient reuse of the hardware may be obtained by sharing the FHT blocks between the inner and the outer decoder.

The complexity of the scheme turns out to be favorable when compared with that of schemes providing similar decoding thresholds and low error floors [10], [16], in the very low coding rate regime. We consider next the MR-LDPC codes [10], assuming the same overall coding rate $\log _{2} q / 3 q$ and field order $q$ w.r.t. the orthogonal case. This turns in the use of a $\left(q / \log _{q}, 1\right)$ MR inner code over $\mathbb{F}_{q}$, whose binary image is a $(q, \log q)$ binary linear code. We have that while the mother LDPC code can be decoded with $\mathcal{O}(q \log q)$ complexity, the inner MR code soft decoding requires in general the correlation of $q$ sequences of length- $q$ bits each. Thus, the soft decoding of inner code has a complexity $\mathcal{O}\left(q^{2}\right)$. Consider next a symbol-based turbo code from [16]. Here, the outer code is based on trellises with $q$ edges emanating from each of the $q$ states. Thus, even if the inner (bi-) orthogonal code can be decoded with complexity $\mathcal{O}(q \log q)$, the outer code decoding has complexity $\mathcal{O}\left(q^{2}\right)$.

\section{B. Achieving Higher Rates}

An intrinsic drawback of the use of Hadamard and first order RM codes as inner codes in the concatenated scheme is the lack of flexibility in the choice of the coding rate, for a fixed outer coding rate. In fact, when a Hadamard code tailored to a field order $q$ is used, the overall coding rate is $R=(1 / 3) \times\left(\log _{2} q / q\right)$, whereas, if a first order RM code is adopted, $R=(1 / 3) \times\left(2 \log _{2} q / q\right)$. Thus, the use of large field orders, which leads to turbo codes with excellent performance [11], turns in extremely-low coding rates. As an example, if $q=2^{8}$, the scheme based on an inner Hadamard code has a coding rate $R=1 / 96$, which is doubled if an inner first order RM code is used. Next we propose a generic framework to provide more flexibility in the overall code rate. Observe that the mapping $C_{1}(\beta): \mathbb{F}_{q} \mapsto \mathscr{C}_{1}$ does not need to be bijective. If we allow different symbols to be represented by the same inner code word, higher coding rates are achieved. The result can be obtained by placing a linear $\left(n_{\mathrm{P}}, k_{\mathrm{P}}\right)$ precode $\mathscr{C}_{\mathrm{P}}$ between the outer and the inner code. The role of the precode is to match the outer code field order with the inner code dimension. Hence, $k_{\mathrm{P}}=m$ and $n_{\mathrm{P}}=k_{1}$. By selecting a precode with rate $R_{\mathrm{P}}=k_{\mathrm{P}} / n_{\mathrm{P}}>1$, the inner code can be a Hadamard/RM code of dimension $k_{1}<m$. Note that the overall rate of the scheme is given by $R=R_{\mathrm{O}} \times R_{\mathrm{P}} \times R_{\mathrm{l}}$. The precode may simply match the outer code field order with the inner code dimension by puncturing selected bits of the binary image of each outer code symbol. This is the case that will be considered in the following.

\section{PERformance}

The performance of non-binary turbo codes with (bi-) orthogonal signal sets is analyzed by Monte Carlo simulations. The code design targets the short length regime $(k<200$ bits). Figure 3 compares the performance of turbo codes over $\mathbb{F}_{64}$, as well as $\mathbb{F}_{256}$ and orthogonal modulation with those of the IS-95(A) standard. For the later, two decoding strategies are considered. In the first case, the inner code is decoded via bit-wise maximum a posteriori (MAP) decoding, providing soft values at the input of the outer Viterbi decoder. In the second case, an iterative (IT) turbo decoding scheme is employed, where the inner and the outer decoders exchange soft information, allowing a gain of nearly $0.5 \mathrm{~dB}$ [12]. All codes have input block size $k=192$ bits. The code on $\mathbb{F}_{64}$ possesses an overall rate $R=1 / 32$, thus it is comparable with the IS-95(A) scheme. At a bit error rate (BER) of $10^{-4}$, the proposed scheme gains roughly $2.5 \mathrm{~dB}$ on the IS-95(A) with IT decoding. The rate $R=1 / 96 \mathbb{F}_{256}$ scheme gains $2.8 \mathrm{~dB}$. Note that the gap between the Shannon limits for rates $1 / 96$ and $1 / 32$ is nearly $0.03 \mathrm{~dB}$. Thus, the gain of the $R=1 / 96$ is quite remarkable. As a confirmation of this, on the same chart the SPB [9] for the continuous-input AWGN channel is provided, with an input block of $k=192$ bits. The $R=1 / 96$ code performs within $0.8 \mathrm{~dB}$ from the corresponding SPB down to a codeword error rate (CER) of $10^{-6}$. The $R=1 / 32$ code over $\mathbb{F}_{64}$ loses nearly $1.2 \mathrm{~dB}$ w.r.t. the $\mathrm{SPB}$ at $\mathrm{CER} \simeq 10^{-5}$.

Figure 4 compares the performance of the same codes in the noncoherent setting. Here, the large gain w.r.t. the IS-95(A) scheme is preserved for both the $\mathbb{F}_{64}$ and $\mathbb{F}_{256}$ turbo codes. However, as already observed by the DE analysis, the higher field order pays back in robustness, whereas the $\mathbb{F}_{64}$ shows a larger degradation w.r.t. the coherent case.

The performance of several codes with an information block size of $k=192$ bits is depicted in Figure 5. In addition to the above-introduced codes, a rate $1 / 48$ code has been obtained by replacing the $(256,8)$ Hadamard code with a $(128,8)$ RM code, whereas a rate $1 / 24$ code has been achieved by concatenating the outer $\mathbb{F}_{256}$ turbo code with an inner $(64,7)$ RM code. Here, the matching between the turbo code field order and inner code dimension has been obtained by puncturing the last bit of the binary representation of each turbo code symbol. The CER for a rate 1/15 superorthogonal turbo code with input block size of 200 bits from [7] under coherent detection is also provided, together with the SPB for each coding rate. Remarkably, the superorthogonal turbo code exhibits an error floor at $C E R \simeq 10^{-4}$, whereas for all codes designed on $\mathbb{F}_{256}$ no sign of error floor is visible down to $\mathrm{CER} \simeq 10^{-6}$. 


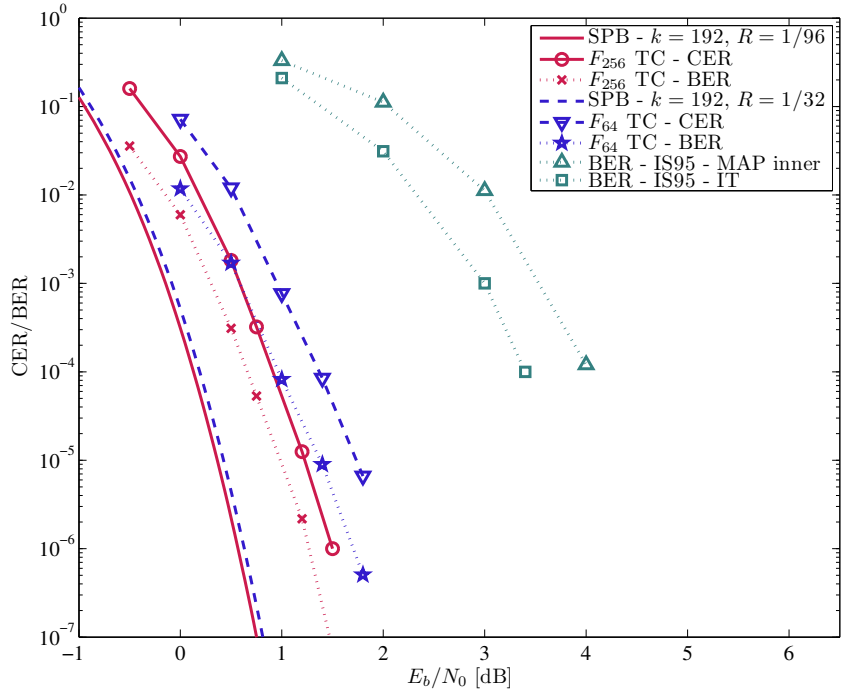

Fig. 3. CER and BER for low-rate turbo codes over $\mathbb{F}_{64}$ and $\mathbb{F}_{256}$ with orthogonal modulation and coherent detection. The BER of the IS95(A) uplink scheme is provided as reference. Information block of 192 bits.

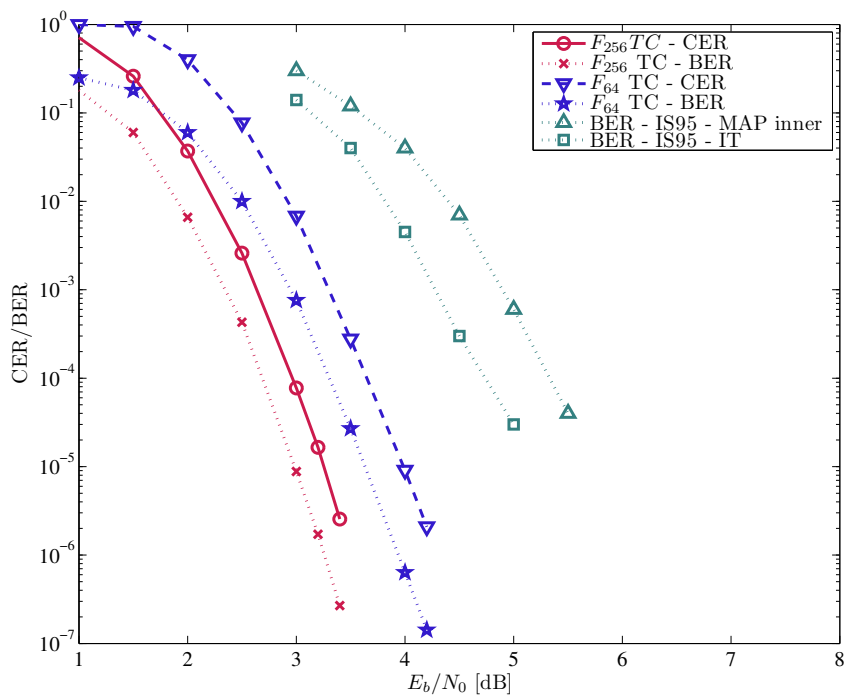

Fig. 4. CER and BER for low-rate turbo codes over $\mathbb{F}_{64}$ and $\mathbb{F}_{256}$ with orthogonal modulation and noncoherent detection. The BER of the IS95(A) up-link scheme is provided as reference. Information block of 192 bits.

\section{CONCLUSIONS}

This paper investigates the design of non-binary turbo codes in concatenation with inner linear block codes for very low coding rates. A DE analysis has been provided for coherent and the noncoherent detection, showing decoding thresholds close to the Shannon limit. When the inner codes are chosen to be Hadamard or first order RM codes a simple decoder implementation is possible, which employs FHTs for decoding both the inner and the outer code. Codeword error rates close within $0.8 \mathrm{~dB}$ from the SPB have been obtained for the proposed schemes, while no floors have been detected down to error rates as low as $C E R \simeq 10^{-6}$.

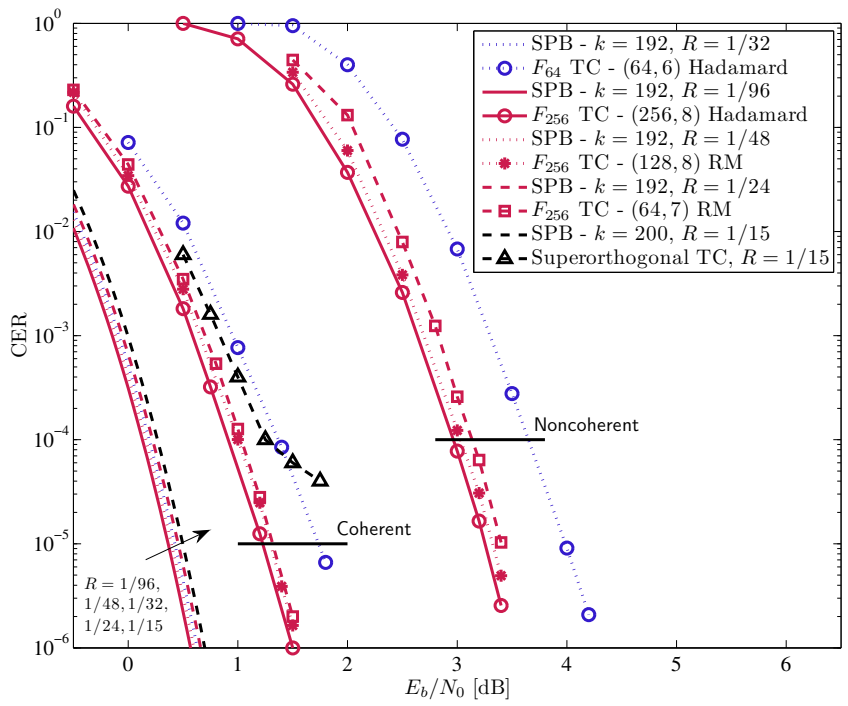

Fig. 5. CER for several low-rate codes over $\mathbb{F}_{64}$ and $\mathbb{F}_{256}$, with information block of 192 bits. Coherent and noncoherent detection.

\section{REFERENCES}

[1] A. J. Viterbi, "Spread spectrum communications-Myths and realities," IEEE Commun. Mag., vol. 17, no. 3, pp. 11-18, 1979.

[2] — CDMA: Principles of Spread Spectrum Communication. Redwood City, CA, USA: Addison Wesley Longman Publishing, 1995.

[3] R. Gallager, Principles of digital communication. Cambridge University Press, 2008.

[4] A. J. Viterbi, "Orthogonal tree codes for communication in the presence of white Gaussian noise," IEEE Trans. Commun., vol. 15, no. 2, pp. 238-242, 1967.

[5] _ " "Very low rate convolution codes for maximum theoretical performance of spread-spectrum multiple-access channels," IEEE J. Sel. Areas Commun., vol. 8, no. 4, pp. 641-649, May 1990.

[6] L. Ping, W. Leung, and K. Wu, "Low-rate turbo-Hadamard codes," IEEE Trans. Inf. Theory, vol. 49, no. 12, pp. 3213-3224, 2003.

[7] P. Komulainen and K. Pehkonen, "Performance evaluation of superorthogonal turbo codes in AWGN and flat Rayleigh fading channels," IEEE J. Sel. Areas Commun., vol. 16, no. 2, pp. 196-205, 1998.

[8] G. Liva, W. E. Ryan, and M. Chiani, "Quasi-cyclic generalized LDPC codes with low error floors," IEEE Trans. Commun., vol. 56, no. 1, pp. 49-57, Jan. 2008.

[9] C. Shannon, "Probability of error for optimal codes in a Gaussian channel," Bell System Tech. J., vol. 38, pp. 611-656, 1959.

[10] K. Kasai, D. Declercq, C. Poulliat, and K. Sakaniwa, "Multiplicatively repeated nonbinary LDPC codes," IEEE Trans. Inf. Theory, vol. 57, no. 10, pp. 6788-6795, 2011.

[11] G. Liva, E. Paolini, S. Scalise, and M. Chiani, "Turbo codes based on time-variant memory- 1 convolutional codes over $\mathbb{F}_{q}$," in Proc. IEEE Int. Conf. on Communications, Kyoto, Japan, Jun. 2011.

[12] R. Herzog, A. Schmidbauer, and J. Hagenauer, "Iterative decoding and despreading improves CDMA-systems using M-ary orthogonal modulation and FEC," in Proc. IEEE Int. Conf. on Communications, Montreal, Canada, 1997, pp. 909-913.

[13] M. Peleg and S. Shamai, "On the capacity of the blockwise incoherent MPSK channel," IEEE Trans. Commun., vol. 46, no. 5, pp. 603-609, May 1998.

[14] J. Berkmann and C. Weiss, "On dualizing trellis-based APP decoding algorithms," IEEE Trans. Commun., vol. 50, no. 11, pp. 1743 - 1757, Nov. 2002.

[15] G. D. Forney Jr., Concatenated Codes. Cambridge, MA: M.I.T. Press, 1966.

[16] M. Bingeman and A. Khandani, "Symbol-based turbo codes," IEEE Commun. Lett., vol. 3, no. 10, pp. 285-287, 1999.

[17] T. Helleseth and $\varnothing$. Ytrehus, "How to find a $[33,8,14]$ code," Univ. of Bergen, Bergen, Norway, Report in Informatics 41, 1989.

[18] F. Mac Williams and N. Sloane, The Theory of Error-Correcting Codes. North Holland Mathematical Libray, 1977. 